\title{
Accelerated Long Term Forgetting in patients with focal seizures: Incidence rate and contributing factors
}

Laurie A. Miller (a),(b), Annu Mothakunnel (b),(c), Emma Flanagan (c),(d), Armin Nikpour (a),(b), Zoe Thayer (a),(b)

(a) Institute of Clinical Neurosciences, Royal Prince Alfred Hospital, and Central Medical School, University of Sydney, Camperdown, NSW, Australia

(b) ARC-Centre of Excellence in Cognition and Its Disorders, University of Sydney, NSW, Australia

(c) Neuroscience Research Australia, Sydney, NSW, Australia

(d) Norwich Medical School, University of East Anglia, Norwich, United Kingdom

\begin{abstract}
Background: Accelerated Long Term Forgetting (ALF) is usually defined as a memory impairment that is seen only at long delays (e.g., after days or weeks) and not at shorter delays (e.g., $30 \mathrm{~min}$ ) typically used in clinical settings. Research indicates that ALF occurs in some patients with epilepsy, but the incidence rates and underlying causes have not been established. In this study, we considered these issues.

Methods: Forty-four patients with a history of focal seizures were tested at $30 \mathrm{~min}$ and 7 day delays for material from the Rey Auditory Verbal Learning Test (RAVLT) and Aggie Figures Test. Recently published norms from a matched group of 60 control subjects (Miller et al., 2015 ) were used to determine whether patients demonstrated ALF, impairment at 30 min or intact memory performance. Results: The incidence of ALF in the epilepsy patients (18\%) was N 3 times higher than normal on the RAVLT, but no different (7\%) from the incidence in normal subjects on the Aggie Figures. A different, but again significantly high, proportion of patients (36\%) showed shorter-term memory deficits on at least one task. ALF was found mainly in patients with temporal-lobe epilepsy, but also occurred in one patient with an extra temporal seizure focus. Presence of a hippocampal lesion was the main predicting factor of ALF.

Conclusions: Many patients with a focal seizure disorder show memory deficits after longer delays that are not evident on standard assessment. The present study explored the factors associated with this ALF memory profile. These new findings will enhance clinical practice, particularly the management of patients with memory complaints.
\end{abstract}

\section{Introduction}

Accelerated Long Term Forgetting (ALF) is a relatively newly de- scribed memory disorder in which a person shows good retention over a short period (e.g., up to $30 \mathrm{~min}$ ), but then forgets at a rapid rate over the next few days or weeks [2,3]. This phenomenon has most often been reported in patients with temporal-lobe epilepsy [4,5] or transient epileptic amnesia [6,7]. Given the nature of standard memory testing, which typically involves shorter delays only, this type of longer- term memory disorder has gone largely undetected in clinical practice until recently. It is important to identify ALF, however, because it may be the first sign of a neurological problem [8] and because subjective memory complaints tend to correlate with long-term retention rather than with short-term scores $[9,10]$. It is not yet clear what proportion of patients with focal seizures have ALF, because research results have generally been presented for individual case studies or as an average for a group. Understanding the incidence of ALF in focal epilepsy and factors that predict its occurrence would improve clinical perspective and potentially offer new insights into longer term memory processes. Many studies of ALF have employed a learning-to-criterion technique to ensure equivalence of 
encoding across subjects. Under these conditions, patients often show intact memory over the initial, $20-30$ min delay interval, but then demonstrate significant loss after days or weeks $[4,9,11]$. That is, as a group, their mean scores are indicative of ALF. This finding of relatively good memory at shorter delays, however, seems at odds with numerous previous reports of memory difficulties at these same, short delays in patients with temporal-lobe epilepsy [12-14], in particular when the hippocampus has been re- moved or is sclerotic [15-17]. It may be that this inconsistency in memory findings at 20-30 min delays is related to differences in learning condition (i.e., learned-to-criterion conditions of the more recent ALF studies versus limited exposure during learning in most of the earlier re- ports). We found support for this proposal when we compared these two learning conditions for stories; ALF was more evident when epilepsy patients had learned the material to criterion than when they heard the stories only once [18]. For the present study, we chose to use standardized memory measures that involve multiple (5) presentations during learning. Recall was then tested at $30 \mathrm{~min}$ and 7 day delays. A recent review paper [19] identified several clinical factors associated with ALF. Interictal discharges are common in patients with ALF, though there is less evidence that seizures during the delay interval [9,20], side of epileptic focus [21-25] or underlying etiology [19] are influential. Site of brain abnormality is probably also important. Most patients identified as showing ALF have had abnormalities in the temporal lobe, though a few recent studies have indicated that patients with an extra temporal focus (i.e., one outside the temporal lobe) can also demonstrate ALF [18,26,27].

It was also noted that patients who present with complaints consistent with ALF and are found to have an epileptic condition (usually TEA) tend to be middle aged or older [19]. It will be interesting to determine whether patients with focal seizures who show ALF are older than those without ALF and/or whether they had a later age of seizure onset.

Because patients with epilepsy can be treated with a number of different antiepileptic drugs (AEDs) alone or in combination, researchers have generally been able to consider only whether the number of AEDs predicts ALF and this has not been found to be the case $[9,20,26]$. Whether any particular medications are more likely than others to cause ALF has yet to be determined.

Structural hippocampal abnormality has been linked both with deficient memory at short delays $(20-30 \mathrm{~min})[15,21,22]$ and with memory decay over $24 \mathrm{~h}[11,18]$. After intervals of days or weeks, however, impairments in memory have not been found to be limited to patients with hippocampal lesions $[10,18,26,28,29]$. A comparison of the impact of a hippocampal lesion at short vs long term retention intervals will help elucidate its contribution to memory over time.

Given that memory for different types of material decays at different rates [7,30,31], incidence of ALF might also be affected by the type of to- be-remembered material. Although most previous studies have detected steeper long-term forgetting rates in people with focal seizures compared to control subjects for both visual and verbal materials [19], visual and verbal tasks have often not been well-matched. With this in mind, the present investigation used 15-item lists consisting of verbal (words) and nonverbal (abstract drawings) material learned and recalled under similar conditions. Earlier work indicated that healthy subjects learn a similar number of items from the two lists [32] and subsequently show similar recall scores for words and drawings at $30 \mathrm{~min}$ and 7 day delays [1]. We will now discover whether patients with focal seizures show similar forgetting rates for the two types of material. By investigating memory task performance at short and long delays, we will determine what proportion of patients with focal seizures show ALF. The associated clinical and demographic characteristics will also be identified.

\section{Methods}

\subsection{Ethics and consent}

This study was approved by Royal Prince Alfred Hospital's Human Research Ethics Committee. All participants provided written informed consent. 


\subsection{Participants}

\subsubsection{Patients}

Potential patients were approached to participate if they met the following inclusion criteria: (1) they were identified by A.N. (Clinical Neurologist) as having a focal seizure disorder on the basis of a full clinical work-up (i.e., EEG, neuroimaging, clinical history); (2) they spoke English; and (3) they had no neurological or psychiatric history other than epilepsy. After testing, any subjects with an estimated Full Scale IQ below the average range (i.e., b 80) were excluded.

Based on clinical interview, age of epilepsy onset, time since onset and frequency of seizures were established. Number and types of AEDs were also recorded. Fifty-one patients were tested initially but 6 failed to complete the testing after a 7-day delay. One additional subject was excluded because her estimated Full Scale IQ fell below 80. Twenty- two male and 22 female participants comprised the final group of 44. A.N. (blind to the neuropsychological results) determined the side and site of epileptic focus as well as the presence of a hippocampal lesion $(\mathrm{n}=17)$.

One patient who had evidence of both left- and right-sided abnormalities was excluded from analyses comparing effects of lesion side. Three patients with temporal plus extra temporal abnormalities were not included in the analyses comparing effects of lesion site.

\subsubsection{Normal control (NC) subjects}

The group of 60 subjects (without neurological or psychiatric history) from our normative study served as a comparison group [1].

\subsection{Neuropsychological test materials}

\subsubsection{Rey Auditory Verbal Learning Test (RAVLT) [33]}

A 15-item word list (List A) is presented over 5 learning trials. In each trial, the list is read aloud to the subject and they are asked to recall as many words as possible. A distractor list of 15 new items (List B) is then read by the examiner and recalled by the subject in a similar fashion (but only once), and an unprompted ("immediate") recall of List A follows. Thirty minutes later, the delayed recall of List A is requested. For this study, subjects were also called 7 days later (without warning) and long term recall of List A was collected over the telephone. The number of words remembered after 30 min served as the 30 min Recall Score and the Percent Change Score was calculated as: [(number recalled at $30 \mathrm{~min}-$ number recalled at 7 days) / recall at $30 \mathrm{~min}] \times 100$.

\subsubsection{Aggie Figures [32].}

This test of memory for a set of 15 abstract line-drawings was created to be a visual analogue of the RAVLT and is presented in a similar fashion. Each line-drawing of List A is shown to the subject for a few seconds, one after another, using a ring-bound booklet. After seeing all 15 items, subjects are provided with a blank sheet of paper and asked to draw as many as possible. This is repeated for 5 learning trials, and then a distractor list (List B) of 15 new drawings is presented and recalled. Immediate and 30 min delayed recall of List A follows. At the time of the phone call seven days later, subjects were instructed to draw again all the figures they could remember and then post back the sheet. Subjects had not been forewarned that their recall would be retested. The number of figures remembered after $30 \mathrm{~min}$ served as the $30 \mathrm{~min}$ Recall Score and the Percent Change Score was calculated as: [(number recalled at $30 \mathrm{~min}-$ number recalled at 7 days $) /$ recall at $30 \mathrm{~min}] \times 100$.

\subsubsection{Test of Premorbid Functioning (TOPF) [34]}


The TOPF is a measure of reading pronunciation ability for words that have irregular grapheme-to-phoneme translation. The number correct is converted to an estimate of Full Scale Intelligence Quotient (FSIQ) on the Wechsler Adult Intelligence Scale IV using age-adjusted norms.

\subsubsection{Depression Anxiety Stress Scale (DASS-21) [35]}

This is a 21-item self-report questionnaire measuring symptom frequency in the last week with regard to depression, anxiety and stress. Upon completion, scores are doubled to match the original 42-item version on which the norms are based. Only the score for the depression symptoms items (DASS-D) was used in this study. A score of 10 or higher suggests at least mild depression.

\section{4. $\quad$ Procedure}

Each patient was tested individually in the Neuropsychology Unit of Royal Prince Alfred Hospital. The four measures described above were administered in the context of a more complete neuropsychological assessment. Each 30 min delay interval was filled with other tests. One week later, subjects were contacted by telephone for the final delayed recalls of the RAVLT and Aggie Figures.

\subsection{Statistical analyses}

Contingency tables were used to compare groups in the proportion of memory profiles. Fisher exact tests were used to test significance because some of the expected cell sizes were b 5. Only results with $\mathrm{p} \mathrm{b} .05$ are reported as significant.

\section{Results}

\subsection{Demographic and clinical characteristics of the groups}

Patients did not differ on demographic or clinical variables when di- vided by either side or site of epileptic focus (see Table 1). With regard to seizure frequency, a little over half (55\%) of the patients had active epilepsy (i.e., $\mathrm{N} 1$ seizure per year). NC subjects did not differ from the patient group in sex distribution ( 29 male, 31 female), mean age $(40.7, \mathrm{SD}=13.4)$ or mean estimated premorbid IQ score $(105.2, \mathrm{SD}=10.9)$.

\subsection{Incidence of memory deficits}

Based on norms in Miller et al. [1], we divided the patients into three groups: 1) NORMAL MEMORY - those with memory scores within nor- mal limits at both the $30 \mathrm{~min}$ and 7 day delays (i.e., both 30 min Recall and Percent Change scores fell within 1.64 SD of the normal mean); 2) DEFICIT BY 30 MIN — those with a 30 min Recall score falling more than 1.64 SD below the normal mean; 3) ALF - those with a normal 30 min Recall score (i.e., within 1.64 SD of the normal mean), but a Per- cent Change in their memory 7 days later more than 1.64 SD above the normal mean (i.e., significantly greater than normal percentage lost).

The incidence rates for patients divided in this way are presented in Fig. 1. First, it is clear that with these fairly conservative cut-off scores (1.64 SD = less than the 5th percentile), many patients performed within normal limits (22/44 on RAVLT; 31/44 on Aggie Figures). Second, Task seemed to affect the incidence rate of a memory deficit; patients were more likely to show normal memory for the Aggie figures than the RAVLT word list, but this was not significant (two-tailed test, $\mathrm{p}=.08$ ). When delayed memory performance on the two tasks was com- pared, patients showed 
slightly higher rates of impairment on the words versus the figures by $30 \min (14 / 44$ vs 10/44) and in terms of ALF ( $8 / 44$ vs 3/44), but neither of these differences reached significance.



Fig. 1. Proportion of epilepsy patients falling into different memory groups.

The incidence of ALF for the patients differed from the incidence rate in the NC group only for the word list (one-tailed test, $\mathrm{p} \mathrm{b}$.05). In the NC group, 3/60 (5\%) showed ALF on the words and 4/60 (7\%) showed it for the figures. When the incidence of a deficit by $30 \mathrm{~min}$ on each task (middle bars of Fig. 1) was compared to the performance of the $\mathrm{NC}$ group, this was found to be significantly higher for the patient group for the RAVLT (one-tailed test, $\mathrm{p} \mathrm{b} .01$ ), but not on the Aggie Figures.

The proportion of subjects who showed ALF on either task was nearly double for the patients compared to the control group (10/44 [23\%] vs 7/60 [12\%], respectively), however this difference was not significant. Only one patient showed ALF on both tasks. There was a more marked difference between patients and NCs in the incidence of a memory deficit by 30 min on either task (16/44 [36\%] vs $10 / 60$ [17\%], respectively) (one tailed test, $\mathrm{p}$ b .05).

When we divided patients into those with active (N 1 seizure per year) versus inactive $(\leq 1$ seizure per year) epilepsy, we found no significant differences in the proportion who showed memory deficits at either delay. Most of the patients with ALF had active epilepsy (7/10 [70\%]), but this did not differ significantly from the proportion with active epilepsy who did not show ALF (17/34 [50\%]).

\subsection{Effect of hippocampal lesion}

A higher proportion of patients with a hippocampal lesion showed memory deficits at both short and long delays, though this difference was more evident for ALF than for early memory deficits (Fig. 2). Of the individual comparisons (the two groups compared at each delay and on each task), only the comparison for ALF on Aggie Figures reached significance (one-tailed test, $\mathrm{p}$ b .05). There were not enough patients with hippocampal lesions to subdivide the group by side for analyses, but we noted that there were similar numbers with left- and right-sided hippocampal abnormalities showing ALF on both tasks.

\begin{tabular}{|c|c|c|c|c|}
\hline Demographic or clinical variable & Left & Right & Temporal & Extra temporal \\
\hline Epilepsy condition: active/inactive (n) & $13 / 9$ & $10 / 11$ & $15 / 12$ & $6 / 8$ \\
\hline Age Mean (SD) & $42.5(12.4)$ & $42.7(12.4)$ & 43.9 (11.1) & $42.0(14.9)$ \\
\hline Estimated IQ Mean (SD) & $100.5(13.1)$ & $103.0(14.0)$ & $101.3(9.5)$ & $103.5(18.0)$ \\
\hline Age of epil onset (yrs) Mean (SD) & $24.7(13.6)$ & $25.6(10.8)$ & $24.0(12.9)$ & $26.4(12.2)$ \\
\hline Number of AEDsMean (SD) & $2.0(1.0)$ & $1.8(.8)$ & $1.9(1.0)$ & $1.8(.9)$ \\
\hline
\end{tabular}


As can be seen in Fig. 3, if we consider whether deficits occurred on either task, then significant differences related to the presence of a hippocampal lesion were evident for both ALF and 30 min recall. Of the patients with a hippocampal lesion, 7/17 (41\%) had ALF on at least one of the two measures, whereas only $3 / 27(11 \%)$ patients without a hippocampal lesion showed ALF (twotailed test, $\mathrm{p} \mathrm{b}$.05). The proportion of patients with a hippocampal lesion showing ALF was also significantly higher than the proportion of NC subjects showing ALF (two-tailed test, $\mathrm{p} \mathrm{b}$.05), but the proportion of patients without hippocampal lesions who showed ALF did not differ from the proportion of NCs with ALF (7/60 [12\%]). With respect to differences in the proportion showing a deficit at $30 \mathrm{~min}$, the comparison between patient groups (i.e., with and without hippocampal lesions) was not significant. There was a significant difference between NCs (10/60 [17\%]) and patients with a hippocampal lesion (7/17 [41\%]) in the proportion who showed a deficit on at least one task by 30 min (one-tailed test, $\mathrm{p} \mathrm{b} .05$ ). A similar comparison between NCs and patients without hippocampal lesions $(9 / 27[33 \%])$ in incidence of deficits by $30 \mathrm{~min}$ recall did not reach significance.

\section{4. $\quad$ Side and site of focus effects}

With respect to frequency distributions, there were no significant differences when site and side of epileptic focus were considered (Table 2).

\subsection{The impact of other clinical and demographic factors on ALF}

T tests were used to compare patients with- to those without-ALF on a number of clinical and demographic measures (Table 3). No significant group differences were found, though mean age of onset was nearly 10 years higher in those with ALF and showed a medium effect size. This suggested that with a few more subjects, this difference would probably reach significance. With respect to AEDs, the two groups did not differ in the mean number taken. The 10 patients with ALF were taking a number of different medications, but the most common one was Lamotrigine ( 7 were taking this). The proportion of patients with ALF who were taking Lamotrigine (7/10 [70\%]) was significantly higher than the proportion without ALF who were taking Lamotrigine (10/34 [29\%]) (two-tailed test, $\mathrm{p} \mathrm{b}$.05). However, it is important to note that there were a high proportion of patients on Lamotrigine who did not show ALF (10/17 [59\%]).

\section{Discussion}

This is one of the first studies to examine incidence rate of ALF in patients with focal epilepsy and to consider its underlying predicting factors. We applied recently established norms [1] to investigate this memory phenomenon for both visual and verbal materials.

\section{RAVLT}

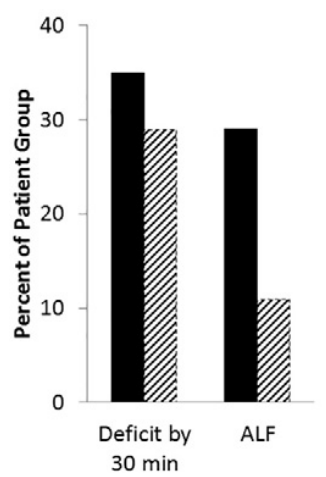

\section{Aggie Figures}

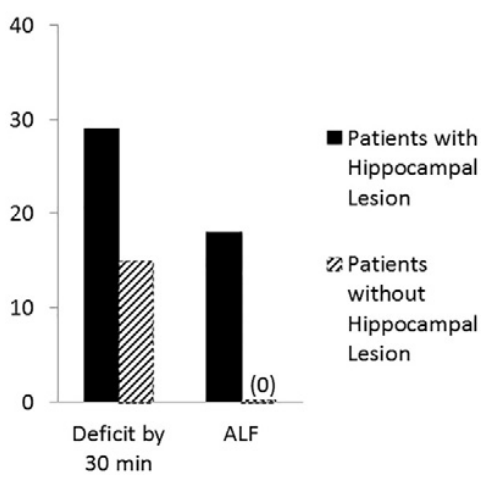


We found that $23 \%$ of the patients with a history of focal seizures showed normal memory initially, but significant loss over a 7-day interval (ALF) on at least one of the two tasks compared to only $12 \%$ of controls. ALF was seen most often in patients with temporal-lobe epilepsy (33\%), though it also occurred in $7 \%$ of patients with an extra temporal focus. Patients with hippocampal lesions were more likely to show ALF (41\%) than those with no structural hippocampal abnormalities $(11 \%)$. Consistent with earlier reports, memory deficits at the 30 min delay were also commonly found in patients with focal epilepsy ( $36 \%$ with a deficit on at least one of the two measures).

Although ALF was seen in some temporal lobe patients without hippocampal lesions and even in one patient with extra temporal epilepsy, our results indicated that ALF is most commonly present in patients with temporal-lobe epilepsy involving the hippocampus. This is consis- tent with the fact that most of the literature on ALF has focused on pa- tients with temporal-lobe epilepsy [19]. Because we collected data only at 30 min and 7 day delays, it was not possible to determine the pattern of decay within that week-long period, but previous work indi- cates that ALF as a consequence of a hippocampal lesion is most evident within $24 \mathrm{~h}[11,26]$. The presence of a hippocampal lesion was also as- sociated with memory deficits at $30 \mathrm{~min}$. Whether hippocampal lesions differ in extent or location in the group with memory impairments in the early stages (i.e., by 30 min) versus the group with ALF has yet to be determined.

Few other clinical or demographic factors were found to influence the incidence rate of ALF. Consistent with previous findings $[9,20,26,36]$, the number of anticonvulsant medications was not found to be re- lated to ALF. In our sample, a high proportion of the patients with ALF were found to be taking Lamotrigine, however, it must be acknowl- edged that this is a popularly used AED and many patients on Lamotrigine did not show ALF. Like others [22], we found no relationship between side of epileptic focus and the presence of ALF. In spite of a prediction raised in the introduction, there was no evidence of a re- lationship between the presence of ALF and a patient's current age, however, there was a trend to suggest that patients with ALF had a later age of epilepsy onset, which is consistent with some previous observa- tions [19]. As we did not assess whether patients experienced seizures within the 7-day delay period, we were unable to examine whether seizure activity during the delay period may have contributed to ALF. How- ever, we found no difference in yearly seizure frequency between patients with and without ALF.

Table 2

Percentage of patients who fell into each category, divided by side and site of seizure focus.

\begin{tabular}{lllllll}
\hline Category & \multicolumn{2}{l}{ Side } & & & \multicolumn{2}{l}{ Site } \\
\cline { 2 - 3 } \cline { 6 - 7 } \cline { 5 - 6 } & Left & Right & & Temporal & Extra temporal \\
\hline Percent impaired at 30 min on RAVLT & 41 & 19 & & 37 & 29 \\
Percent impaired at 30 min on Aggie Figures & 18 & 29 & & 26 & 21 \\
Percent impaired at 30 min on either task & 41 & 29 & & 41 & 36 \\
Percent with ALF on RAVLT & 18 & 19 & & 26 & 7 \\
Percent with ALF on Aggie Figures & 9 & 10 & & 11 & 7 \\
Percent showing ALF on either task & 27 & 19 & & 33 & 7 \\
\hline
\end{tabular}

Table 3

Comparing patients with $(n=10)$ and without $(n=34)$ ALF on clinical, demographic and neuropsychological measures.

\begin{tabular}{llll}
\hline Variable & With ALF Mean & Without ALF & \multicolumn{1}{c}{$\begin{array}{l}\text { T test result (with } \\
\text { Cohen's d) }\end{array}$} \\
\hline Age & $48.1(8.9)$ & Mean (SD) & $\mathrm{t}=-1.6, p=.12 ; \mathrm{d}=.63$ \\
Estimated IQ & $102.1(9.0)$ & $41.2(12.7)$ & $\mathrm{t}=-.10, p=.98 ; \mathrm{d}=.06$ \\
Age of onset & $31.2(16.5)$ & $101.0(14.5)$ & $\mathrm{t}=-1.9, p=.06 ; \mathrm{d}=.60$ \\
Time since onset & $16.9(17.9)$ & $23.0(10.1)$ & $\mathrm{t}=.24, p=.81 ; \mathrm{d}=.08$ \\
Number of AEDs & $2.1(1.2)$ & $18.2(14.0)$ & $\mathrm{t}=-1.0, p=.32 ; \mathrm{d}=.29$ \\
Seizure frequency (per year) & $48.7(112.9)$ & $1.8(.8)$ & $\mathrm{t}=.53, p=.60 ; \mathrm{d}=.17$ \\
DASS depression & $12.6(8.5)$ & $32.5(73.4)$ & $\mathrm{t}=-1.6, p=.12 ; \mathrm{d}=.55$ \\
\hline
\end{tabular}


Rates of ALF differed on our two tasks (18\% for RAVLT, 7\% for Aggie Figures) and the percentage of patients showing ALF was significantly higher only for words. Similarly, Narayanan and colleagues [30] found ALF for verbal material (RAVLT) and not visual material (Rey Complex Figure) when mean memory scores for a group of patients with temporal-lobe epilepsy were contrasted to those of a NC group. There would seem to be a few possible reasons for these types of results. Word-list memory has been found to be more susceptible to disruption by focal epileptic discharges than memory for abstract drawings over a four day period [19]. Also, Davies et al. [37] linked impairments in word retrieval to hippocampal damage. Either of these clinical factors might have resulted in poorer long term retrieval of the RAVLT word list com- pared to the Aggie Figures. In addition, it is known that factors such as novelty, modality (verbal versus nonverbal) and style of presentation (seen vs heard) can affect learning and short term retention [38]. Whether all of these are important in determining longer term memory remains to be explored, but it was clear that task material affects the chances of ALF being demonstrated by patients with focal epilepsy.

The importance of considering the degree of early learning in studies of ALF was pointed out by Elliott et al. [39] as well as Butler and Zeman [40]. Indeed, it seems reasonable to propose that a task with a higher initial learning score (in this case, the RAVLT) is more likely to reveal a significant drop in memory than a task with a lower initial learning score. Arguing against this proposal, however, was the finding that per- cent retention between $30 \mathrm{~min}$ and 7 days was the same for NC subjects on the 2 tasks, irrespective of the 2-point difference in their learning by the 5th trial. Also, if anything, by 30 min delay, patients were scoring slightly higher on the Aggie Figures than the RAVLT, so when their ALF score is considered, according to this proposal, there should be more chance of showing ALF for the Aggie Figures. Hence, degree of initial learning does not seem to be the factor predicting which task will reveal ALF.

In contrast to the present results, Bell et al., [14,41] found no evidence of ALF for verbal material in patients with temporal-lobe epilepsy using similar stimuli and learning conditions to the ones employed here. Instead, Bell [14] found a high incidence of memory impairment (more than - 1 SD from the normal mean) in the patients at short delays. There are several possible differences that might explain the contrasting results. First, the percentage of patients in the Bell studies with active epilepsy (44\%) was lower than that in ours. As it has previously been established that epileptiform activity is associated with ALF $[9,19]$, the higher proportion of patients with active epilepsy in our sample might have resulted in higher levels of ALF. Consistent with this, we found that patients with ALF in our study tended to have active epilepsy. Second, it is possible that a smaller percentage of the patients in Bell's studies had significant hippocampal lesions. A substantial percentage of their patients (26\%) had not had an MRI and although there was a significant difference between the scanned patients and the control group in mean hippocampal volume, patients were not classified as having (or not having) a significant hippocampal abnormality. We might have had a higher proportion of patients with significant hippocampal lesions in our sample to explain the higher level of ALF. Third, Bell and colleagues use a generous definition of "impairment" (i.e., more than -1 SD from the normal mean). With this definition, a much higher percentage of the epilepsy patients are found to be im- paired at $30 \mathrm{~min}$ and therefore will fail to meet the criteria for ALF. Using a cut-off at the 5th percentile (more than 1.64 SD from normal) we ensured that only patients who did quite poorly were classified as having an early or late memory deficit. Fourth, subtle differences in the material, learning paradigms or long-term delays might explain the different rates of ALF. Further investigation of these intriguing differences in rates of ALF could be used to elucidate long term memory processes.

In this study, we defined ALF as a deficit that showed up only after a 7-day delay. This was so that we could determine how many patients show only longer-term memory deficits, which would have been missed in standard clinical practice. In fact, when we broadened the definition of ALF to look for patients who showed excessively steep de- clines in memory from the $30 \mathrm{~min}$ to 7 day delays, irrespective of whether they showed a deficit at the standard delay interval, we found that ALF was almost twice as likely to be identified. Most patients who showed a deficit at the standard (30 min) delay also showed a significantly steep decline between 30 min and 7 days. 
Overall, it is important to note that a patient with ALF on one task does not necessarily demonstrate it on others. In this study, only one patient showed ALF on both types of material. Further investigation of the impact of type of to-be-remembered material and other methodological factors is needed, as the current study employed only two measures of ALF. Conclusions from this study are also limited to those with partial epilepsy, as those with primary generalized epilepsy were excluded.

There seems to be an emerging consensus that consideration of ALF is an important part of the clinical profile of patients with epilepsy. Memory performance at very long delays correlates better with subjective complaints than memory performance at standard delays $[9,10]$ and ALF may be the first presentation of a neurological problem [8]. The likelihood of finding ALF will be influenced by many factors, including the number of tasks used to detect it, definition of "impairment", type of to-be-remembered material and characteristics of the patient sample (e.g., especially proportion with hippocampal damage and pro- portion with active epilepsy). The results from the present study indicate that testing memory for a word list after a one-week interval is particularly useful in detecting ALF in patients with focal epilepsy.

\section{Conflict of Interest}

LAM has received funding from UCB Pharma in the form of an educational grant to run memory training programs and honoraria from this company to speak at neurological conferences. AN has received research funding from UCB Pharma and honoraria from EISAI and SciGen. There are no other conflicts of interest.

\section{Acknowledgements}

The study was funded in part by the ARC Centre of Excellence Grant CE110001021 in Cognition and Its Disorders. We thank the participants who donated their time to this project.

\section{References}

[1] Miller LA, Flanagan E, Mothakunnel A, Mohamed A, Thayer Z. Old dogs with new tricks: detecting accelerated long-term forgetting by extending traditional measures. Epilepsy Behav 2015;45:205-11.

[2] Manes F, Graham KS, Zeman A, de Lujan Calcagno M, Hodges JR. Autobiographical amnesia and accelerated forgetting in transient epileptic amnesia. J Neurol Neurosurg Psychiatry 2005;76:1387-91.

[3] Kapur N, Scholey K, Moore E, Barker S, Brice J, Thompson S, et al. Long-term reten- tion deficits in two cases of disproportionate retrograde amnesia. $\mathrm{J}$ Cogn Neurosci 1996;8:416-35.

[4] Blake RV, Wroe SJ, Breen EK, McCarthy RA. Accelerated forgetting in patients with epilepsy: evidence for an impairment in memory consolidation. Brain 2000;123: 472-83.

[5] Mameniskiene R, Jatuzis D, Kaubrys G, Budrys V. The decay of memory between delayed and long-term recall in patients with temporal lobe epilepsy. Epilepsy Behav 2006;8:278-88.

[6] Butler CR, Graham KS, Hodges JR, Kapur N, Wardlaw JM, Zeman AZ. The syndrome of transient epileptic amnesia. Ann Neurol 2007;61:587-98.

[7] Muhlert N, Milton F, Butler CR, Zeman AZ. Accelerated forgetting of real-life events in transient epileptic amnesia. Neuropsychologia 2010;48:3235-44.

[8] Hornberger M, Mohamed A, Miller L, Watson J, Thayer Z, Hodges JR. Focal retrograde amnesia: extending the clinical syndrome of transient epileptic amnesia. J Clin Neurosci 2010;17:1319-21. 
[9] Fitzgerald Z, Thayer Z, Mohamed A, Miller LA. Examining factors related to accelerated long-term forgetting in epilepsy using ambulatory EEG monitoring. Epilepsia 2013;54:819-27.

[10] Butler CR, Bhaduri A, Acosta-Cabronero J, Nestor PJ, Kapur N, Graham KS, et al. Transient epileptic amnesia: regional brain atrophy and its relationship to memory deficits. Brain 2009;132:357-68.

[11] Lah S, Mohamed A, Thayer Z, Miller L, Diamond K. Accelerated long-term forgetting of verbal information in unilateral temporal lobe epilepsy: is it related to structural hippocampal abnormalities and/or incomplete learning? J Clin Exp Neuropsychol 2014;36:158-69.

[12] Milner B. Disorders of learning and memory after temporal lobe lesions in man. Clin Neurosurg 1972;19:421-46.

[13] Hermann BP, Wyler AR, Richey ET, Rea JM. Memory function and verbal-learning ability in patients with complex partial seizures of temporal-lobe origin. Epilepsia 1987;28:547-54.

[14] Bell B. WMS-III logical memory performance after a two-week delay in temporal lobe epilepsy and control groups. J Clin Exp Neuropsychol 2006;28:1435-43.

[15] Miller LA, Munoz DG, Finmore M. Hippocampal sclerosis and human memory. Arch Neurol 1993;50:391-4.

[16] Hermann B, Wyler A, Somes G, Berry A, Dohan F. Pathological status of the mesial temporal lobe predicts memory outcome from left anterior temporal lobectomy. Neurosurgery 1992;31:652-6.

[17] Jones-Gotman M, Zatorre RJ, Olivier A, Andermann F, Cendes F, Staunton H, et al. Learning and retention of words and designs following excision from medial or lateral temporal-lobe structures. Neuropsychologia 1997;35:963-73.

[18] Ricci M, Mohamed A, Savage G, Miller LA. Disruption of learning and long-term retention of prose passages in patients with focal epilepsy. Epilepsy Behav 2015;51: 104-11.

[19] Fitzgerald Z, Mohamed A, Ricci M, Thayer Z, Miller L. Accelerated long-term forget- ting: a newly identified memory impairment in epilepsy. J Clin Neurosci 2013;20: 1486-91.

[20]

Muhlert N, Grunewald RA, Hunkin NM, Reuber M, Howell S, Reynders H, et al. Accelerated long-term forgetting in temporal lobe but not idiopathic generalised epilepsy. Neuropsychologia 2011;49:2417-26.

[21] Bengner T, Malina T, Lindenau M, Voges B, Goebell E, Stodieck S. Face memory in MRI-positive and MRI-negative temporal lobe epilepsy. Epilepsia 2006;47:1904-14.

[22] Wilkinson H, Holdstock JS, Baker G, Herbert A, Clague F, Downes JJ. Long-term accel-erated forgetting of verbal and non-verbal information in temporal lobe epilepsy. Cortex 2011;48:317-32.

[23] Kapur N, Millar J, Colbourn C, Abbott P, Kennedy P, Docherty T. Very long-term amnesia in association with temporal lobe epilepsy: evidence for multiple-stage consolidation processes. Brain Cogn 1997;35:58-70.

[24] Martin RC, Loring DW, Meador KJ, Lee GP, Thrash N, Arena JG. Impaired longterm retention despite normal verbal learning in patients with temporal lobe dysfunction.

Neuropsychology 1991;5:3-12.

[25] Helmstaedter C, Hauff M, Elger CE. Ecological validity of list-learning on a selective reminding procedure and self-reported memory in healthy individuals and those with temporal lobe epilepsy. J Clin Exp Neuropsychol 1998;20:365-75.

[26] Ricci M, Mohamed A, Savage G, Boserio J, Miller LA. The impact of epileptiform ab- normalities and hippocampal lesions on retention of recent autobiographical experiences: adding insult to injury? Neuropsychologia 2015;66:259-66.

[27] Lah S, Mohamed A, Thayer Z, Miller L, Diamond K. Accelerated long-term forgetting of verbal information in patients with temporal and extratemporal partial epilepsy. $\mathrm{J}$ Int Neuropsychol Soc 2012;18:64-5. 
[28] Butler C, Kapur N, Zeman A, Weller R, Connelly A. Epilepsy-related long-term amnesia: anatomical perspectives. Neuropsychologia 2012;50:2973-80.

[29] Dayan E, Cohen LG. Neuroplasticity subserving motor skill learning. Neuron 2011; 72:443-54.

[30] Narayanan J, Duncan R, Greene J, Leach J-P, Razvi S, McLean J, et al. Accelerated long-term forgetting in temporal lobe epilepsy: verbal, nonverbal and autobiographical memory. Epilepsy Behav 2012;25:622-30.

[31] Tramoni E, Felician O, Barbeau EJ, Guedj E, Guye M, Bartolomei F, et al. Long-term consolidation of declarative memory: insight from temporal lobe epilepsy. Brain 2011;134:816-31.

[32] Majdan A, Sziklas V, Jones-Gotman M. Performance of healthy subjects and patients with resection from the anterior temporal lobe on matched tests of verbal and visuoperceptual learning. J Clin Exp Neuropsychol 1996;18:416-30.

[33] Rey A. L'examen psychologique dans les cas d'encephalopathie traumatique. Arch Psychol 1941;28:286-340.

[34] Wechsler D. Test of premorbid functioning. San Antonio: The Psychological Corporation; 2009.

[35] Lovibond SH, Lovibond PF. Manual for the depression anxiety stress scales. 2nd ed. Psychology Foundation: Sydney; 1995.

[36] Trachtenberg JT, Chen BE, Knott GW, Feng GP, Sanes JR, Welker E, et al. Longterm in vivo imaging of experience-dependent synaptic plasticity in adult cortex. Nature 2002;420:788-94.

[37] Davies K, Bell B, Bush A, Hermann B, Dohan F, Jaap A. Naming decline after left anterior temporal lobectomy. Correlates with pathological status of resected hippocampus. Epilepsia 1998;39:407-19.

[38] Redoblado MA, Grayson SJ, Miller LA. Lateralized-temporal-lobe-lesion effects on learning and memory: examining the contributions of stimulus novelty and presentation mode. $\mathrm{J}$ Clin Exp Neuropsychol 2003;25:36-48.

[39] Elliott G, Isaac CL, Muhlert N. Measuring forgetting: a critical review of accelerated long-term forgetting studies. Cortex 2014;54:16-32.

[40] Butler CR, Zeman AZ. Recent insights into the impairment of memory in epilepsy: transient epileptic amnesia, accelerated long-term forgetting and remote memory impairment. Brain : a journal of neurology 2008;131:2243-63.

[41] Bell B, Fine J, Dow C, Seidenberg M, Hermann B. Temporal lobe epilepsy and the se- lective reminding test: the conventional 30-minute delay suffices. Psychol Assess 2005;17:103-9. 